\title{
Tanning Attitudes and Behaviors in Adolescents and Young Adults
}

\author{
Daniel C. Glade, MD; Austin C. Smith, MD; Valerie T. Fisher-Shiu, MD; Robert T. Gilson, MD
}

\section{PRACTICE POINTS}

- Dermatologists are the preferred educators of skin care for adolescents and young adults.

- Social media is an underused medium for skin cancer prevention education and can reach those who do not regularly see a dermatologist.

- Education of young people focusing on their concerns about maintaining a youthful appearance instead of the possibility of developing skin cancer in the future might be more effective.

Skin cancer rates have been steadily increasing over the last 20 years despite persistent efforts to educate the public on skin cancer prevention and sun safety. Adolescents and young adults are an especially important demographic to reach, as increased UV exposure during these years leads to greatly increased risks of developing skin cancer. Our survey aims to investigate the attitudes and behaviors regarding sun protection and tanning among adolescents and young adults (age range, 13-27 years).

Cutis. 2021;107:261-263.

ntentional tanning-through sun exposure and tanning beds-is an easily avoidable contributor to skin cancer development and an important area for public education. Since the advent of social media, a correlation between social media use and increased indoor tanning behaviors has been reported. ${ }^{1}$ In 2010, $11.3 \%$ of US adults aged 18 to 29 years reported using a tanning bed in the last 12 months. ${ }^{2}$ The American Academy of Dermatology first published their "Position Statement on Indoor Tanning" in 1998, endorsing a ban on the sale of indoor tanning equipment for nonmedical purposes. ${ }^{3}$

Although there has been no outright ban on indoor tanning, regulations have been put in place in many states-including Texas, where (as of 2013) a person younger than 18 years must have written consent from their parent(s) to use a tanning bed. Despite efforts of organizations including the American Academy of Dermatology and the government to educate the public on skin cancer prevention and sun safety, the skin cancer rate has been steadily increasing over the last 20 years.

There is a constant campaign among dermatologists to educate their patients on how to reduce or avoid the risk for skin cancer, including the use of sunscreen and avoidance of tanning. Adolescents and young adults are an especially important demographic to reach and educate because increased UV light exposure during these years leads to a greatly increased risk for skin cancer later in life. ${ }^{4}$ Data on the overall prevalence of tanning and the demographics of participation in tanning activities are important to capture and can be used to efficiently target higher-risk populations.

In this study, we aimed to investigate the attitudes and behaviors of adolescents and young adults regarding sun protection and tanning. We also aimed to determine which avenues, including social media, would be most effective at educating about skin cancer awareness and sun protection to the higher-risk younger population.

\section{Materials and Methods}

We developed an institutional review board-approved protocol for the prospective collection of data from registered patients at the dermatology clinic of the

From the University of Texas Health at San Antonio, Long School of Medicine. Drs. Fisher-Shiu and Gilson are from the Division of Dermatology. The authors report no conflict of interest.

Correspondence: Daniel C. Glade, MD, Department of Dermatology, University of Texas Health San Antonio, 7979 Wurzbach Rd, San Antonio, TX78220 (gladed@uthscsa.edu).

doi:10.12788/cutis.0246 
Mays Cancer Center at the University of Texas Health at San Antonio. A paper survey containing 15 rating-scale questions was administered to 60 patients aged 13 to 27 years. Surveys were administered during intake, prior to the patients' visit with a dermatologist; all visits were of a functional (not cosmetic) nature. Data collection spanned June to August 2018. Survey results were entered into Research Electronic Data Capture (REDCap) software for qualitative analysis.

\section{Results}

Sixty patients responded to the survey. The mean age of respondents was 19.5 years. No surveys were excluded from the data set. Table 1 provides baseline characteristics of respondents. Some respondents left questions unanswered, resulting in questions with fewer than 60 responses.

Among respondents to the survey, $70 \%(42 / 60)$ reported it is very important to protect their skin from sun exposure, and 30\% (18/60) reported it is somewhat important. Regarding sunscreen use, 70\% (42/60) indicated they use sunscreen only before outdoor activities, $12 \%(7 / 60)$ use sunscreen daily, and 17\% (10/60) never use sunscreen. Of those who use sunscreen, 52\% (28/54) do so to prevent skin damage and aging and 44\% (24/54) to prevent skin cancer. Twenty-three percent (13/56) of

\section{TABLE 1. Baseline Characteristics of Respondents}

\begin{tabular}{ll}
\hline Characteristic & Responses, $\mathrm{n}(\%)$ \\
\hline Mender & $20(33.3)$ \\
\hline Female & $40(66.7)$ \\
Race ${ }^{a}$ & $36(60.0)$ \\
White & $8(13.3)$ \\
\hline Black/African American & $9(15.0)$ \\
\hline Asian & $10(16.7)$ \\
\hline Other/unkown & $19(31.7)$ \\
\hline Ethnicitya & $15(25.0)$ \\
\hline Non-Spanish & $20(33.3)$ \\
\hline Mexican & $7(11.7)$ \\
\hline Spanish/Hispanic/Latino & \\
\hline Other & \\
\hline
\end{tabular}

apatients might have had $>1$ response. respondents reported finding tanned skin attractive; $26 \%(14 / 55)$ reported wanting to be tan. Looking at race, 28\% (10/36) of Whites, 25\% (5/20) of Spanish/ Hispanic/Latinos, and 22\% (2/9) of Asians found tanned skin attractive; no Black respondents found tanned skin attractive.

Regarding tanning, 12\% (7/57) reported using a tanning bed in their lifetime and $4 \%(2 / 57)$ in the last year; $34 \%(19 / 56)$ reported deliberately tanning outdoors; and $9 \%(5 / 56)$ reported using sunless or spray-on tanning. Dermatologists (75\% [42/56]), primary care physicians $(69.6 \%$ [39/56]), and parents (46.4\% [26/56]) were perceived as more effective sources of skin care education; among media modalities, television (33.9\% [19/56]), Instagram (30.4\% [17/60]), and YouTube (23.2\% [13/60]) were perceived as more effective sources of skin care education (Table 2).

\section{Comment}

Perceptions of Tanning-Almost one-quarter of respondents found tanned skin attractive, which might reflect a shift from prior generations. Compared to the $11 \%$ of respondents in the 2010 survey, $^{2}$ only 3.5\% (2/57) of our respondents reported using a tanning bed in the last year, which could reflect the results of recent Texas legislation restricting the use of tanning beds by adolescents.

\section{TABLE 2. Responses to: "What would be the most effective way to receive information about skin care?"}

\begin{tabular}{ll}
\hline Modality & $\begin{array}{l}\text { Responses, } \mathbf{n}(\%)^{\mathrm{a}} \\
(\mathbf{n}=56)\end{array}$ \\
\hline Dermatologist & $42(75.0)$ \\
\hline Primary care physician & $39(69.6)$ \\
\hline Parent & $26(46.4)$ \\
\hline Television & $19(33.9)$ \\
\hline Instagram & $17(30.4)$ \\
\hline YouTube & $13(23.2)$ \\
\hline Magazines & $12(21.4)$ \\
\hline Twitter & $11(19.6)$ \\
\hline Snapchat & $11(19.6)$ \\
\hline Facebook & $9(16.1)$ \\
\hline Teacher & $8(14.3)$ \\
\hline Pinterest & $4(7.1)$ \\
\hline Patients mist have & 11 modaty. \\
\hline
\end{tabular}

apatients might have selected $>1$ modality. 
An alarming number of respondents reported going outdoors with the intention of tanning; although it appears that indoor tanning education has been successful, this finding shows that there is still a need for sun protection education because outdoor tanning is not a suitable alternative. A small number of respondents reported getting a sunless or spray-on tan, which is a risk-free alternative to indoor tanning.

Despite all respondents stating that protecting skin from the sun is important, most respondents surveyed do not use sunscreen daily. More respondents use sunscreen to prevent damage and aging than to prevent skin cancer. Young people might be more alarmed by the threat of early aging and losing their "youthful appearance" than by the possibility of developing skin cancer in the distant future. This discrepancy might indicate a lack of knowledge and be an important focus for future education efforts.

Perceptions of Trustworthiness of Education SourcesOur findings show dermatologists and primary care physicians are important educators on skin protection. Primary care physicians should remain vigilant to recognize at-risk patients who would benefit from skin protection education, especially those who do not see a dermatologist. Education of young people focusing on their concern over maintaining a youthful appearance instead of the possibility of developing skin cancer in the future might be more effective.

Although education provided by a physician is effective, using media-particularly social media-might be more efficient. Television, Instagram, and YouTube were listed by respondents as the 3 most preferred media outlets for skin health education, which shows important areas of focus for future advertising. Facebook was listed at a surprisingly low level, possibly showing the change in use of certain social media websites among this age group. According to the Pew Research Center, the most widely used social media apps among young adults aged 18 to 29 years are YouTube (91\%), Facebook $(63 \%)$, Instagram (67\%), and Snapchat (62\%). More than half of the same demographic visit Facebook (74\%),
Instagram (63\%), Snapchat (61\%), and YouTube (51\%) daily. ${ }^{5}$ Although respondents to our survey were not specifically asked about the frequency of their use of social media and our data set includes patients younger than 18 years, we know that social media use has been increasing over the last decade among adolescents. ${ }^{1}$ Therefore, we assume that more than one-half of respondents to our survey use their reported social media platforms daily.

Social media is an underused medium for skin cancer prevention education and can reach those who do not regularly see a dermatologist. Unlike printed pamphlets and posters, advertisements through social media can use metrics such as age, race, gender, and interests to target high-risk individuals.

Study Limitations-This was a single-site study of currently enrolled dermatology patients who might be more aware of skin protection than the general population because they are being treated by a dermatologist. Survey questions, regarding demographics, required by our institution, could not effectively differentiate Hispanic and White patients. Respondents could have been subject to the Hawthorne effect-awareness that their behavior is being observed-when responding to the survey because it was administered in the office prior to being seen by a dermatologist.

\section{REFERENCES}

1. Falzone AE, Brindis CD, Chren M-M, et al. Teens, tweets, and tanning beds: rethinking the use of social media for skin cancer prevention. Am J Prev Med. 2017;53(3 suppl 1):S86-S94.

2. Centers for Disease Control and Prevention. Use of indoor tanning devices by adults-United States, 2010. MMWR Morb Mortal Wkly Rep. 2012;61:323-326

3. American Academy of Dermatology. Position statement on indoor tanning. Amended November 14, 2009. Accessed January 10, 2021. https://server.aad.org/Forms/Policies/Uploads/PS/PS-Indoor $\% 20$ Tanning\%2011-16-09.pdf?

4. American Academy of Dermatology. Indoor tanning. Accessed January 10, 2020. https://www.aad.org/media/stats-indoor-tanning

5. Perrin A, Anderson M. Share of U.S. adults using social media, including Facebook, is mostly unchanged since 2018. Pew Research Center; April 10, 2019. Accessed April 16, 2021. https://www.pewresearch.org /fact-tank/2019/04/10/share-of-u-s-adults-using-social-media -including-facebook-is-mostly-unchanged-since-2018/ 